\title{
Staticity and regularity for zero rest-mass fields near spatial infinity on flat spacetime
}

\author{
E. Gasperín*1 and J. A. Valiente Kroon ${ }^{\dagger, 2}$ \\ ${ }^{1}$ CENTRA, Departamento de Física, Instituto Superior Técnico IST, Universidade de Lisboa UL, \\ Avenida Rovisco Pais 1, 1049 Lisboa, Portugal \\ ${ }^{1 \mathrm{a}}$ Institut de Mathématiques de Bourgogne (IMB), UMR 5584, CNRS, Université de Bourgogne \\ Franche-Comté, F-21000 Dijon, France. \\ ${ }^{2}$ School of Mathematical Sciences, Queen Mary, University of London, Mile End Road, London E1 4NS, \\ United Kingdom.
}

July 28, 2021

\begin{abstract}
Linear zero-rest-mass fields generically develop logarithmic singularities at the critical sets where spatial infinity meets null infinity. Friedrich's representation of spatial infinity is ideally suited to study this phenomenon. These logarithmic singularities are an obstruction to the smoothness of the zero-rest-mass field at null infinity and, in particular, to peeling. In the case of the spin-2 field it has been shown that these logarithmic singularities can be precluded if the initial data for the field satisfies a certain regularity condition involving the vanishing, at spatial infinity, of a certain spinor (the linearised Cotton spinor) and its totally symmetrised derivatives. In this article we investigate the relation between this regularity condition and the staticity of the spin-2 field. It is shown that while any static spin-2 field satisfies the regularity condition, not every solution satisfying the regularity condition is static. This result is in contrast with what happens in the case of General Relativity where staticity in a neighbourhood of spatial infinity and the smoothness of the field at future and past null infinities are much more closely related.
\end{abstract}

Keywords: Conformal methods, spinors, staticity, cylinder at spatial infinity, regularity.

PACS: 04.20.Ex, 04.20.Ha, 04.20.Gz

\section{Introduction}

Obtaining a satisfactory understanding of the consequences of the degeneracy of the conformal structure of spacetime at spatial infinity is one of the key open problems in the mathematical theory of isolated systems in General Relativity - the so-called problem of spatial infinity. A programme to systematically investigate this problem was initiated with the seminal work of Friedrich in [7. The key idea of this work is the development of a representation of spatial infinity (the cylinder at spatial infinity) which allows the formulation of a regular Cauchy problem in a neighbourhood of spatial infinity for the conformal Einstein field equations. This framework allows to show that, unless the initial data is fine-tuned, the solutions to the conformal Einstein field equations (and in particular the components of the rescaled Weyl tensor) develop logarithmic

\footnotetext{
*E-mail address:edgar.gasperi@tecnico.ulisboa.pt

$\dagger$ E-mail address:j.a.valiente-kroon@qmul.ac.uk
} 
singularities at the critical sets where null infinity meets spatial infinity. In the particular case of time-symmetric initial data sets for the Einstein field equations which admit a point compactification at infinity for which the resulting conformal metric is analytic then its possible to show that a certain subset of the logarithmic singularities can be avoided if the conformal metric $\boldsymbol{h}$ satisfies the (conformally invariant) condition

$$
D_{\left\{i_{p} \cdots i_{1}\right.} b_{j k\}}(i)=0, \quad p=0,1,2, \ldots,
$$

where $b_{i j}$ denotes the Cotton-Bach tensor of the metric $\boldsymbol{h}$ and $\{\cdots\}$ denotes the operation of computing the symmetric tracefree part - in particular, if $\boldsymbol{h}$ is conformally flat then $b_{j k}=0$. Observe that this condition is imposed at the point at infinity of the asymptotic end. Accordingly, it is a truly asymptotic condition. The regularity condition (10) has also arisen in slightly different contexts and is satisfied by static initial data sets - see [5, 2]. Subsequent analysis has shown that although condition (1) is a necessary condition for avoiding logarithmic singularities at the critical sets, it is by no means sufficient - see [28, 27. Static solutions to the Einstein field equations have been shown to be logarithmic free at the critical points of Friedrich's representation of spatial infinity [9. Moreover, the analysis in [33, 31] strongly suggests the conjecture that, among the class of time symmetric initial data sets, only those which are static in a neighbourhood of infinity will give rise to developments which are free of logarithmic singularities at the critical sets - see also [10. The gluing techniques developed in [4, 3] allow the construction of large classes of initial data sets with this property. The interested reader is directed to [1] for further discussion of this extensive topic - see also 32, Chapter 21.

Generically, linearised fields propagating on the Minkowski spacetime also develop logarithmic singularities at the critical sets - see e.g. [26]. In particular, for the massless spin-2 field, there is an analogue of condition (11) which, in this case, precludes the development of all logarithmic singularities at least for a large class of initial data regular at infinity. This condition is expressed in terms of the totally symmetric linearised Cotton-Bach spinor $B_{A B C D}$ as

$$
D_{\left(A_{p} B_{p}\right.} \cdots D_{A_{1} B_{1}} B_{A B C D)}(i)=0, \quad p=0,1,2, \ldots,
$$

where $D_{A B}$ denotes the spinorial counterpart of the 3-dimensional Levi-Civita connection $D_{i}$. The spinor $B_{A B C D}$ is essentially the curl of the physical spin-2 field.

Given the strong relation between the regularity condition (1) and the staticity of the associated spacetime, it is natural to ask whether there exists a connection between condition (2) and static spin-2 fields - i.e. spin-2 fields whose Lie derivative with respect to the static Killing vector of the Minkowski spacetime vanish. This is the question addressed in this article. Our analysis shows that while the regularity condition (2) is satisfied by any such static spin-2 fields, not every spin-2 field arising from initial data for which condition (2) holds is static. More precisely, the spin-2 field will be static if and only if

$$
B_{A B C D}=0 \quad \text { on the initial hypersurface }
$$

- see Theorem 1 In particular, $B_{A B C D}$ and its derivatives to all orders vanish at $i$. Accordingly, the requirement of smoothness at null infinity is a far less stringent requirement for the linearised field than for the (non-linear) gravitational field. This observation should serve as a caution towards extracting conclusions about asymptotics of the gravitational field from an analysis of of the linearised equations.

\section{Outline of the article}

Section 2 provides a brief overview of Friedrich's representation of spatial infinity in terms of a cylinder. Section 3 provides a discussion of the properties of the spin- 2 field which are relevant for the analysis in this article. Section 4 contains a brief overview of the space-spinor formalism used to analyse the spin-2 field. Section 5 provides a study of static spin-2 fields. 


\section{Notations and Conventions}

The signature convention for (Lorentzian) spacetime metrics will be $(+,-,-,-)$. In the rest of this article $\{a, b, c, \ldots\}$ denote abstract tensor indices and $\{\boldsymbol{a}, \boldsymbol{b}, \boldsymbol{c}, \ldots\}$ will be used as spacetime frame indices taking the values $0, \ldots, 3$. In this way, given a basis $\left\{\boldsymbol{e}_{\boldsymbol{a}}\right\}$ a generic tensor is denoted by $T_{a b}$ while its components in the given basis are denoted by $T_{\boldsymbol{a} \boldsymbol{b}} \equiv T_{a b} \boldsymbol{e}_{\boldsymbol{a}}{ }^{a} \boldsymbol{e}_{\boldsymbol{b}}{ }^{b}$. Greek indices $\left\{\mu_{\nu},{ }_{\lambda}, \ldots\right\}$ are used to denote spacetime coordinates while $\{\alpha, \beta, \ldots\}$ play the role of spatial coordinate indices. The core of the analysis makes use of spinors. For this, the notation and conventions of Penrose \& Rindler [19] will be followed. In particular, capital Latin indices $\{A, B, C, \ldots\}$ will denote abstract spinor indices while boldface capital Latin indices $\left\{\boldsymbol{A}_{\boldsymbol{B}}, \boldsymbol{C}, \ldots\right\}$ will denote frame spinorial indices with respect to a specified spin dyad $\left\{\delta_{\boldsymbol{A}}{ }^{A}\right\}$. The conventions for the curvature tensors are fixed by the relation

$$
\left(\nabla_{a} \nabla_{b}-\nabla_{b} \nabla_{a}\right) v^{c}=R_{d a b}^{c} v^{d}
$$

\section{Spatial infinity in the Minkowski spacetime}

In this section we provide a quick overview of Friedrich's representation of spatial infinity for the Minkowski spacetime. The reader interested in further details is referred to [7, 8, 26, 32, 1].

Let $\left(\mathbb{R}^{4}, \tilde{\boldsymbol{\eta}}\right)$ denote the Minkowski spacetime and consider Cartesian coordinates $\left(\tilde{x}^{\mu}\right)=\left(\tilde{t}, \tilde{x}^{\alpha}\right)$ in which the Minkowski line element reads

$$
\tilde{\boldsymbol{\eta}}=\eta_{\mu \nu} \mathbf{d} \tilde{x}^{\mu} \otimes \mathbf{d} \tilde{x}^{\nu}, \quad \eta_{\mu \nu} \equiv \operatorname{diag}(1,-1,-1,-1)
$$

Let $\left(\tilde{t}, \tilde{\rho}, \theta^{\mathcal{A}}\right)$ with $\mathcal{A}=2,3$ denote spherical polar coordinates defined by $\tilde{\rho}^{2} \equiv \delta_{\alpha \beta} \tilde{x}^{\alpha} \tilde{x}^{\beta}$ where $\delta_{\alpha \beta} \equiv \operatorname{diag}(1,1,1)$, and $\left(\theta^{\mathcal{A}}\right)$ represents an arbitrary choice of coordinates on $\mathbb{S}^{2}$. In these coordinates the metric $\tilde{\boldsymbol{\eta}}$ is given by

$$
\tilde{\boldsymbol{\eta}}=\mathbf{d} \tilde{t} \otimes \mathbf{d} \tilde{t}-\mathbf{d} \tilde{\rho} \otimes \mathbf{d} \tilde{\rho}-\tilde{\rho}^{2} \boldsymbol{\sigma}
$$

with $\tilde{t} \in(-\infty, \infty), \tilde{\rho} \in[0, \infty)$ and $\boldsymbol{\sigma}$ represents the standard round metric on $\mathbb{S}^{2}$.

\subsection{The basic formalism}

In the sequel we restrict our discussion to the region

$$
\mathcal{D} \equiv\left\{\tilde{x}^{\mu} \mid \eta_{\mu \nu} \tilde{x}^{\mu} \tilde{x}^{\nu}<0\right\}
$$

which contains the asymptotic region of the Minkowski spacetime around spatial infinity. On $\mathcal{D}$ introduce inversion coordinates $\left(x^{\mu}\right)=\left(t, x^{\alpha}\right)$ defined by

$$
x^{\mu}=-\tilde{x}^{\mu} / \tilde{X}^{2}, \quad \tilde{X}^{2} \equiv \eta_{\mu \nu} \tilde{x}^{\mu} \tilde{x}^{\nu}
$$

The inverse transformation is given by

$$
\tilde{x}^{\mu}=-x^{\mu} / X^{2}, \quad X^{2}=\eta_{\mu \nu} x^{\mu} x^{\nu},
$$

with

$$
X^{2}=1 / \tilde{X}^{2}
$$




\subsubsection{The standard representation of spatial infinity}

Rewriting the physical Minkowski metric $\tilde{\boldsymbol{\eta}}$ in these coordinates leads to a conformal representation with conformal metric given by

$$
\bar{\eta}=\Xi^{2} \tilde{\boldsymbol{\eta}}
$$

where

$$
\overline{\boldsymbol{\eta}}=\eta_{\mu \nu} \mathbf{d} x^{\mu} \otimes \mathbf{d} x^{\nu}, \quad \Xi=X^{2} .
$$

Observe that in this representation, the conformal metric $\overline{\boldsymbol{\eta}}$ is, locally the Minkowski metric in particular, the origin of $\left(\mathbb{R}^{4}, \overline{\boldsymbol{\eta}}\right)$ corresponds to the the spatial infinity $i^{0}$ of the Minkowski spacetime $\left(\mathbb{R}^{4}, \tilde{\boldsymbol{\eta}}\right)$. Introducing unphysical polar coordinates $\left(t, \rho, \theta^{\mathcal{A}}\right)$ where $\rho^{2} \equiv \delta_{\alpha \beta} x^{\alpha} x^{\beta}$, the metric $\overline{\boldsymbol{\eta}}$ and conformal factor $\Xi \mathrm{read}$

$$
\overline{\boldsymbol{\eta}}=\mathbf{d} t \otimes \mathbf{d} t-\mathbf{d} \rho \otimes \mathbf{d} \rho-\rho^{2} \boldsymbol{\sigma}, \quad \Xi=t^{2}-\rho^{2},
$$

with $t \in(-\infty, \infty)$ and $\rho \in(0, \infty)$. Unwrapping the above definitions, shows that relation between the physical and unphysical polar coordinates is given by

$$
\tilde{t}=-\frac{t}{t^{2}-\rho^{2}}, \quad \tilde{\rho}=\frac{\rho}{t^{2}-\rho^{2}} .
$$

\subsubsection{Friedrich's representation}

In order to introduce Friedrich's representation of spatial infinity (the so-called F-gauge), one considers a new time coordinate $\tau$ defined via the relation

$$
t=\rho \tau
$$

In terms of this new coordinate the conformal metric $\bar{\eta}$ reads

$$
\overline{\boldsymbol{\eta}}=\rho^{2} \mathbf{d} \tau \otimes \mathbf{d} \tau-\left(1-\tau^{2}\right) \mathbf{d} \rho \otimes \mathbf{d} \rho+\rho \tau(\mathbf{d} \rho \otimes \mathbf{d} \tau+\mathbf{d} \tau \otimes \mathbf{d} \rho)-\rho^{2} \boldsymbol{\sigma} .
$$

The above line element suggests a new conformal representation of the Minkowski spacetime with conformal metric given by

$$
\boldsymbol{\eta} \equiv \frac{1}{\rho^{2}} \overline{\boldsymbol{\eta}}
$$

Writing the metric $\boldsymbol{\eta}$ explicitly one finds that

$$
\boldsymbol{\eta}=\mathbf{d} \tau \otimes \mathbf{d} \tau-\frac{\left(1-\tau^{2}\right)}{\rho} \mathbf{d} \rho \otimes \mathbf{d} \rho+\frac{\tau}{\rho}(\mathbf{d} \rho \otimes \mathbf{d} \tau+\mathbf{d} \tau \otimes \mathbf{d} \rho)-\boldsymbol{\sigma},
$$

which is clearly is singular at $\rho=0$. Notice, however, that its contravariant version

$$
\boldsymbol{\eta}^{\sharp}=\left(1-\tau^{2}\right) \boldsymbol{\partial}_{\tau} \otimes \boldsymbol{\partial}_{\tau}+\rho \tau\left(\boldsymbol{\partial}_{\tau} \otimes \boldsymbol{\partial}_{\rho}+\boldsymbol{\partial}_{\rho} \otimes \boldsymbol{\partial}_{\tau}\right)-\rho^{2} \boldsymbol{\partial}_{\rho} \otimes \boldsymbol{\partial}_{\rho}-\boldsymbol{\sigma}^{\sharp},
$$

is regular — see [1] for further discussion on this peculiarity of Friedrich's representation of spatial infinity. Associated to the above contravariant line element one has a null frame $\left\{\boldsymbol{e}_{\boldsymbol{A} \boldsymbol{A}^{\prime}}\right\}$ such that

$$
\eta^{\sharp}=\epsilon^{A B} \epsilon^{A^{\prime} B^{\prime}} e_{A A^{\prime}} \otimes e_{B B^{\prime}}
$$

with

$$
\begin{aligned}
& \boldsymbol{e}_{\mathbf{0 0 ^ { \prime }}}=\frac{1}{\sqrt{2}}\left((1-\tau) \boldsymbol{\partial}_{\tau}+\rho \boldsymbol{\partial}_{\rho}\right) \\
& \boldsymbol{e}_{\mathbf{1 1 ^ { \prime }}}=\frac{1}{\sqrt{2}}\left((1+\tau) \boldsymbol{\partial}_{\tau}-\rho \boldsymbol{\partial}_{\rho}\right)
\end{aligned}
$$




$$
\begin{aligned}
& e_{01^{\prime}}=\frac{1}{\sqrt{2}} \partial_{+}, \\
& e_{10^{\prime}}=\frac{1}{\sqrt{2}} \partial_{-},
\end{aligned}
$$

where $\left\{\boldsymbol{\partial}_{+}, \partial_{-}\right\}$is a complex null frame on

$$
\mathcal{Q}_{\tau, \rho} \equiv\{p \in \mathcal{M} \mid \tau=\text { const }, \quad \rho=\text { const }\} \approx \mathbb{S}^{2}
$$

satisfying

$$
\left[\boldsymbol{\partial}_{\tau}, \partial_{ \pm}\right]=0, \quad\left[\boldsymbol{\partial}_{\rho}, \partial_{ \pm}\right]=0 .
$$

As discussed in the Appendix of [15, the use of a specific choice of coordinates $\left(\theta^{\mathcal{A}}\right)$ for $\mathbb{S}^{2}$ is not required in the subsequent discussion. Another way to deal with the the deficiencies of spherical coordinates is to lift the structures to a suitable bundle space. This approach is briefly discussed in Section 2.2 .

\subsubsection{The structure of the conformal boundary}

In the conformal representation introduced in the previous section spatial infinity corresponds to the set of points with $\rho=0$ and has the topology of $\mathbb{R} \times \mathbb{S}^{2}$-hence the name of the cylinder at spatial infinity. The rescaling relating the physical Minkowski spacetime $\left(\mathbb{R}^{4}, \tilde{\boldsymbol{\eta}}\right)$ and the cylinder conformal representation $(\mathcal{M}, \boldsymbol{\eta})$ is given by

$$
\boldsymbol{\eta}=\Theta^{2} \tilde{\boldsymbol{\eta}}, \quad \Theta \equiv \rho\left(1-\tau^{2}\right),
$$

and

$$
\mathcal{M} \equiv\left\{p \in \mathbb{R}^{4} \mid-1 \leq \tau(p) \leq 1, \quad \rho(p) \geq 0\right\} .
$$

This representation is closely related to the properties of conformal geodesics - see e.g. 1 . However, this feature will not be exploited in the sequel. The physical Minkowski spacetime corresponds to the region

$$
\tilde{\mathcal{M}} \equiv\{p \in \mathcal{M} \mid-1<\tau(p)<1, \quad \rho(p)>0\},
$$

while future and past null infinity are located at

$$
\begin{aligned}
\mathscr{I}^{+} & \equiv\{p \in \mathcal{M} \mid \tau(p)=1\}, \\
\mathscr{I}^{-} & \equiv\{p \in \mathcal{M} \mid \tau(p)=-1\},
\end{aligned}
$$

and spatial infinity is "blown-up" to a set $\mathcal{I} \approx \mathbb{R} \times \mathbb{S}^{2}$ given by

$$
\mathcal{I} \equiv\{p \in \mathcal{M}|| \tau(p) \mid<1, \rho(p)=0\}, \quad I^{0} \equiv\{p \in \mathcal{M} \mid \tau(p)=0, \rho(p)=0\} .
$$

Moreover, one identifies the critical sets

$$
\begin{aligned}
& \mathcal{I}^{+} \equiv\{p \in \mathcal{M} \mid \tau(p)=1, \rho(p)=0\} \\
& \mathcal{I}^{-} \equiv\{p \in \mathcal{M} \mid \tau(p)=-1, \rho(p)=0\}
\end{aligned}
$$

corresponding to the sets where spatial infinity "touches" null infinity. Additionally, let

$$
\tilde{\mathcal{S}}_{\star}=\left\{p \in \mathbb{R}^{4} \mid \tilde{t}(p)=0\right\}, \quad \mathcal{S}_{\star}=\{p \in \mathcal{M} \mid \tau(p)=0\},
$$

describing the time symmetric hypersurface of the Minkowski spacetime. Observe that $\tilde{\mathcal{S}}_{\star}$ and the interior of $\mathcal{S}_{\star}$ coincide as sets of points. The region where $\mathcal{S}_{\star}$ intersects $\mathcal{I}$ will be denoted as $\mathcal{I}^{0}$. 


\subsection{The fibre space}

As it is well known, the vectors $\boldsymbol{e}_{\mathbf{0 1}} \mathbf{1}^{\prime}$ and $\boldsymbol{e}_{\mathbf{1 0}}$ cannot define non-vanishing smooth vector fields everywhere over a manifold with the topology of $\mathbb{S}^{2}$. In order to deal with this technical difficulty, the equations are lifted to a 5-dimensional submanifold of the bundle of normalised spin frames. The details of this construction and its particularisation to the case of Minkowski spacetime has been detailed in [7. In the following paragraph we provide a brief overview of this construction.

Rotations of the form $\boldsymbol{e}_{\mathbf{0 1}}{ }^{\prime} \mapsto e^{i \vartheta} \boldsymbol{e}_{\mathbf{0 1}}, \vartheta \in \mathbb{R}$, in the planes orthogonal to $\boldsymbol{e}_{\mathbf{0 0}} \mathbf{0}^{\prime}$ and $\boldsymbol{e}_{\mathbf{1 1}}$ leave these submanifolds invariant. Accordingly, it defines a subbundle with structure group $U(1)$ which projects onto $\mathcal{M} \backslash \mathcal{I}$. This projection is given by the Hopf map $S U(2) \rightarrow S U(2) / U(1) \simeq \mathbb{S}^{2}$. All the structures on $\mathcal{M} \backslash \mathcal{I}$ are then lifted to this subbundle. In an abuse of notation, we make use of the same symbols to denote the original objects on $\mathcal{M} \backslash \mathcal{I}$ and their lifted counterparts. On the subbundle we consider coordinates $\tau, \rho$ and $s \in S U(2)$. The lift is carried out in such a way that the lifted fields $\boldsymbol{e}_{\mathbf{0 0}}$ and $\boldsymbol{e}_{\mathbf{1 1}}$ ' have the same coordinate expressions as in equations (9a)-(9b). The coordinate $\rho$ is then extended in a natural manner to include this value. In terms of the coordinates $(\tau, \rho, s)$ on the extended bundle, to be denoted again by $\mathcal{M}$, the the lifted metric and conformal factor are given, again, by (8) and (10). In this context, $\sigma$ denotes the pull-back of the line element on $\mathbb{S}^{2}$ to $S U(2)$. Accordingly, one has that

$$
\mathcal{M} \simeq[-1,1] \times[0, \infty] \times S U(2),
$$

while

$$
\mathcal{I} \simeq[-1,1] \times S U(2), \quad \mathcal{I}^{0} \simeq S U(2), \quad \mathcal{I}^{ \pm} \simeq S U(2), \quad \mathscr{I}^{ \pm} \simeq \mathbb{R} \times S U(2),
$$

are now considered as subsets of the subbundle $\mathcal{M}$.

In order to define vector fields on the $S U(2)$ part of the subbundle, consider the basis

$$
u_{1}=\frac{1}{2}\left(\begin{array}{cc}
0 & \mathrm{i} \\
\mathrm{i} & 0
\end{array}\right), \quad u_{2}=\frac{1}{2}\left(\begin{array}{cc}
0 & -1 \\
1 & 0
\end{array}\right), \quad u_{3}=\frac{1}{2}\left(\begin{array}{cc}
\mathrm{i} & 0 \\
0 & -\mathrm{i}
\end{array}\right)
$$

of the Lie algebra of $S U(2)$ with commutator $\left[u_{i}, u_{j}\right]=\epsilon_{i j k} u_{k}$. Denote by $\mathbf{Z}_{1}, \mathbf{Z}_{2}, \mathbf{Z}_{3}$, the left invariant vector fields generated by $u_{1}, u_{2}, u_{3}$ on the Lie group $S U(2)$. In particular, $\mathbf{Z}_{3}$ is the vertical vector field which generates the group $U(1)$ acting on the fibres of $\mathcal{M}$ and define $\mathbf{X} \equiv-2 \mathbf{i Z}_{3}$. Finally, define the complex conjugate vector fields

$$
\mathbf{X}_{ \pm} \equiv-\left(\mathbf{Z}_{2} \pm \mathrm{i} \mathbf{Z}_{1}\right)
$$

and define

$$
e_{01^{\prime}}=-\frac{1}{\sqrt{2}} \mathbf{X}_{+}, \quad e_{10^{\prime}}=-\frac{1}{\sqrt{2}} \mathbf{X}_{-} .
$$

The construction outline above leads to vector fields

$$
e_{\boldsymbol{A} \boldsymbol{A}^{\prime}}, \quad \mathbf{Z}_{3} \quad \text { on } \quad \mathcal{M} \backslash \mathcal{I}
$$

which extend smoothly to $\mathcal{I}$ and satisfy

$$
\boldsymbol{\eta}\left(\mathbf{Z}_{3}, \cdot\right)=0, \quad \boldsymbol{\eta}\left(\boldsymbol{e}_{\boldsymbol{A} \boldsymbol{A}^{\prime}}, \boldsymbol{e}_{\boldsymbol{B}, \boldsymbol{B}^{\prime}}\right)=\epsilon_{\boldsymbol{A} \boldsymbol{B} \epsilon_{\boldsymbol{A}^{\prime} B^{\prime}}} \quad \text { on } \quad \mathcal{M} \backslash \mathcal{I}
$$

Finally, the connection form induced on $\mathcal{M} \backslash \mathcal{I}$ by the corresponding connection form on the bundle of normalised spin coefficients defines the following non-vanishing connection coefficients with respect to the frame $\left\{\boldsymbol{e}_{\boldsymbol{A} \boldsymbol{A}^{\prime}}\right\}$ :

$$
\Gamma_{00^{\prime} 01}=\Gamma_{11^{\prime} 01}=-\frac{1}{2 \sqrt{2}} .
$$

All the other connection coefficients vanish. 


\section{The spin-2 field equation and the regularity condition}

The interest on the spin-2 equation stems from the fact that it can be used to study the linearised gravitational field and as a model for the Bianchi equations satisfied by the components of the Weyl tensor. In contrast with more traditional approaches to linearised gravity where the key unknown is a metric perturbation, in this case, the linearised gravitational field is encoded in a tensor representing the weak-field Riemann curvature [20, 26]. If the weak-field limit of the vacuum Einstein field equations are imposed, it becomes a traceless tensor (hence with the same symmetries of the Weyl curvature tensor) and can be succinctly described by a totally symmetric spinor $\phi_{A B C D} \rightarrow$ see [20]. The linearised Bianchi equations provide the following spinorial field equations for the field $\phi_{A B C D}$ :

$$
\nabla_{A^{\prime}}{ }^{A} \phi_{A B C D}=0 .
$$

Due to its conformal properties — see Lemma 2 - the spin-2 equation (11) can also be interpreted as a toy model for the conformal Einstein field equations - see [26]. The central point to be discussed in this section is that the spin-2 field $\phi_{A B C D}$ propagating in $(\mathcal{M}, \boldsymbol{\eta})$ has, in general, polyhomogeneous solutions. This was originally shown in [26] in the language of fibre bundles and making use of the fibre space discussed in Subsection 2.2 - 7, 8. A similar analysis of the solutions that avoids the use of this extended bundle space was given in [15] for the calculation of the Newman-Penrose constants. In this section we review part of these constructions and recall the regularity condition found in [26] that controls the appearance - at the level of initial dataof the logarithmic terms in a Taylor like expansion for the components of $\phi_{A B C D}$ close to $i^{0}$.

\subsection{Component expressions}

Let $\left\{\epsilon_{\boldsymbol{A}}{ }^{A}\right\}$ be a spinor dyad with $\epsilon_{\mathbf{0}}{ }^{A}=o^{A}$ and $\epsilon_{\mathbf{1}}{ }^{A}=\iota^{A}$. In terms of this spin dyad, the spinor $\phi_{A B C D}$ is encoded in the following five complex scalars

$$
\begin{gathered}
\phi_{0} \equiv \phi_{A B C D} O^{A} O^{B} O^{C} o^{D}, \quad \phi_{1} \equiv \phi_{A B C D} O^{A} o^{B} O^{C} \iota^{D}, \quad \phi_{2} \equiv \phi_{A B C D} O^{A} O^{B} \iota^{C} \iota^{D}, \\
\phi_{3} \equiv \phi_{A B C D} O^{A} \iota^{B} \iota^{C} \iota^{D}, \quad \phi_{4} \equiv \phi_{A B C D} \iota^{A} \iota^{B} \iota^{C} \iota^{D},
\end{gathered}
$$

which have spin weight $2,1,0,-1,-2$ respectively. In the following we consider coefficients $\phi_{0}, \ldots, \phi_{4}$ as their lift to the fibre space introduced in Section 2.2. A direct calculation shows that the spin-2 equation (11) implies the system of evolution equations

$$
\begin{aligned}
& (1+\tau) \boldsymbol{\partial}_{\tau} \phi_{0}-\rho \boldsymbol{\partial}_{\rho} \phi_{0}+\mathbf{X}_{+} \phi_{1}=-2 \phi_{0} \\
& \boldsymbol{\partial}_{\tau} \phi_{1}+\frac{1}{2} \mathbf{X}_{-} \phi_{0}+\frac{1}{2} \mathbf{X}_{+} \phi_{2}=-\phi_{1} \\
& \boldsymbol{\partial}_{\tau} \phi_{2}+\frac{1}{2} \mathbf{X}_{-} \phi_{1}+\frac{1}{2} \mathbf{X}_{+} \phi_{3}=0 \\
& \boldsymbol{\partial}_{\tau} \phi_{3}+\frac{1}{2} \mathbf{X}_{-} \phi_{2}+\frac{1}{2} \mathbf{X}_{+} \phi_{4}=\phi_{3} \\
& (1-\tau) \boldsymbol{\partial}_{\tau} \phi_{4}+\rho \boldsymbol{\partial}_{\rho} \phi_{4}+\mathbf{X}_{-} \phi_{3}=2 \phi_{4}
\end{aligned}
$$

and constraint equations

$$
\begin{aligned}
& \tau \boldsymbol{\partial}_{\tau} \phi_{1}-\rho \boldsymbol{\partial}_{\rho} \phi_{1}+\frac{1}{2} \mathbf{X}_{+} \phi_{2}-\frac{1}{2} \mathbf{X}_{-} \phi_{0}=0 \\
& \tau \boldsymbol{\partial}_{\tau} \phi_{2}-\rho \boldsymbol{\partial}_{\rho} \phi_{2}+\frac{1}{2} \mathbf{X}_{+} \phi_{3}-\frac{1}{2} \mathbf{X}_{-} \phi_{1}=0 \\
& \tau \boldsymbol{\partial}_{\tau} \phi_{3}-\rho \boldsymbol{\partial}_{\rho} \phi_{3}+\frac{1}{2} \mathbf{X}_{+} \phi_{4}-\frac{1}{2} \mathbf{X}_{-} \phi_{2}=0
\end{aligned}
$$


Remark 1. The above evolution and constraint equations correspond, respectively, to equations (29a)-(29e) and (30a)-(30c) in [15] with the replacement

$$
\check{\partial} \mapsto \mathbf{X}_{+}, \quad \bar{\partial} \mapsto \mathbf{X}_{-},
$$

where $\bar{\partial}$ and $\bar{\partial}$ are the eth and ethbar operators of the Newman-Penrose formalism — see e.g. [19]. Observe, however, that the equations in [15] are defined over the (conformal) spacetime manifold whereas (12a)-112e) and (13a)-(13c) are defined on the fibre space discussed in Section 2.2 .

\subsection{Asymptotic expansions}

The fact that $\phi_{n}$ with $n=0,1,2,3,4$, have a well defined spin weight allows to encode the angular dependence in terms of spin-weighted spherical harmonics - see [22, 15, 26]. When working on the fibre space of Section 2.2 one makes use of the functions $T_{i}{ }_{k}{ }_{k}: S U(2) \rightarrow \mathbb{C}$ introduced in [7] rather than the usual functions ${ }_{s} Y_{l m}$.

In the following, consistent with the discussion in [7, 26, 15] we consider solutions to the equations (12a)-(12e) and (13a)-(13c) of the form

$$
\phi_{n}=\sum_{p=|2-n| \ell=|2-n|}^{\infty} \sum_{k=0}^{p} \frac{1}{p !} a_{n, p ; \ell, m}(\tau) T_{2 p}{ }^{k} \ell-n \rho^{p},
$$

where $a_{n, p ; \ell, m}: \mathbb{R} \rightarrow \mathbb{C}$ and $n=0, \ldots, 4$. The correspondence between the functions $T_{i}{ }^{j} k$ and the harmonics ${ }_{s} Y_{l m}$ is given by

$$
{ }_{s} Y_{n m} \mapsto(-\mathrm{i})^{s+2 n-m} \sqrt{\frac{2 n+1}{4 \pi}} T_{2 n}{ }^{n-m}{ }_{n-s}
$$

- see [12] for more details. The expression (14) is taken as an Ansatz for the solution. The convergence of solutions to the spin-2 equations of the form given by (14) has been analysed in [8. Ansatz (14) allows to reduce the problem of constructing asymptotic expansions to the spin-2 equations to the analysis of a set of ordinary differential equations for $a_{n, p, \ell, m}(\tau)$. To see this, let

$$
\left.\phi_{n}^{(p)} \equiv \frac{\partial^{p} \phi_{n}}{\partial \rho^{p}}\right|_{\rho=0}
$$

with $n=0,1,2,3,4$. Taking the $p$-th derivative of equations (12a)-(13c) respect to $\rho$ and evaluating at the cylinder $\mathcal{I}$ gives the relations

$$
\begin{aligned}
& (1+\tau) \boldsymbol{\partial}_{\tau} \phi_{0}^{(p)}+\mathbf{X}_{+} \phi_{1}^{(p)}+(p-2) \phi_{0}^{(p)}=0 \\
& \boldsymbol{\partial}_{\tau} \phi_{1}^{(p)}+\frac{1}{2} \mathbf{X}_{-} \phi_{0}^{(p)}+\frac{1}{2} \mathbf{X}_{+} \phi_{2}^{(p)}+\phi_{1}^{(p)}=0, \\
& \boldsymbol{\partial}_{\tau} \phi_{2}^{(p)}+\frac{1}{2} \mathbf{X}_{-} \phi_{1}^{(p)}+\frac{1}{2} \mathbf{X}_{+} \phi_{3}^{(p)}=0, \\
& \boldsymbol{\partial}_{\tau} \phi_{3}^{(p)}+\frac{1}{2} \mathbf{X}_{-} \phi_{2}^{(p)}+\frac{1}{2} \mathbf{X}_{+} \phi_{4}^{(p)}-\phi_{3}^{(p)}=0 \\
& (1-\tau) \boldsymbol{\partial}_{\tau} \phi_{4}^{(p)}+\mathbf{X}_{-} \phi_{3}^{(p)}+(p-2) \phi_{4}^{(p)}=0
\end{aligned}
$$

and

$$
\begin{aligned}
& \tau \boldsymbol{\partial}_{\tau} \phi_{1}^{(p)}+\frac{1}{2} \mathbf{X}_{+} \phi_{2}^{(p)}-\frac{1}{2} \mathbf{X}_{-} \phi_{0}^{(p)}-p \phi_{1}^{(p)}=0 \\
& \tau \boldsymbol{\partial}_{\tau} \phi_{2}^{(p)}+\frac{1}{2} \mathbf{X}_{+} \phi_{3}^{(p)}-\frac{1}{2} \mathbf{X}_{-} \phi_{1}^{(p)}-p \phi_{2}^{(p)}=0
\end{aligned}
$$




$$
\tau \boldsymbol{\partial}_{\tau} \phi_{3}^{(p)}+\frac{1}{2} \mathbf{X}_{+} \phi_{4}^{(p)}-\frac{1}{2} \mathbf{X}_{-} \phi_{2}^{(p)}-p \phi_{3}^{(p)}=0
$$

These equations, in turn, upon substitution of the expansion (14), imply a system of ordinary differential equations for $a_{n, p ; \ell, m}$ with $p \geq 2$ and $2 \leq \ell \leq p$. In fact, part of these equations constitutes an algebraic system that allows to determine $a_{1, p, \ell, m}, a_{2, p, \ell, m}$ and $a_{3, p, \ell, m}$, in terms of $a_{0, p, \ell, m}$ and $a_{4, p, \ell, m}$. The problem is then reduced to solve the following system of equations for $a_{0, p, \ell, m}$ and $a_{4, p, \ell, m}$

$$
\begin{aligned}
& \left(1-\tau^{2}\right) \ddot{a}_{0}+(4+2(p-1) \tau) \dot{a}_{0}+(p+\ell)(p-\ell+1) a_{0}=0, \\
& \left(1-\tau^{2}\right) \ddot{a}_{4}+(-4+2(p-1) \tau) \dot{a}_{4}+(p+\ell)(p-\ell+1) a_{4}=0 .
\end{aligned}
$$

Further details of this calculation can be found in [15, 26. Also, observe that if $a_{0}(\tau)$ solves (18a) then $a_{0}^{s}(\tau) \equiv a_{0}(-\tau)$ solves equation (18b). Equations (18a) and 18b are examples of Jacobi differential equations. For $l \neq p$, the solution is given by the so-called Jacobi polynomials as summarised in the following:

Lemma 1. The solutions to the system (18a) and (18b) can be written as

$$
\begin{aligned}
& a_{0, p ; l, m}(\tau)=C_{p, \ell, m} Q_{p, \ell}^{1}(\tau)+(-1)^{\ell} D_{p, \ell, m} Q_{p, \ell}^{3}(\tau), \\
& a_{4, p ; l, m}(\tau)=D_{p, \ell, m} Q_{p, \ell}^{1}(-\tau)+(-1)^{\ell} C_{p, \ell, m} Q_{p, \ell}^{3}(-\tau),
\end{aligned}
$$

with

$$
C_{p, \ell, m} \equiv X_{A}^{-1} a_{0, p ; l, m}(0)+X_{B}^{-1} a_{4, p ; l, m}(0), \quad D_{p, \ell, m} \equiv X_{B}^{-1} a_{0, p ; l, m}(0)+X_{A}^{-1} a_{4, p ; l, m}(0),
$$

where $Q_{p, \ell}^{n}(\tau)$ denotes some not everywhere vanishing Jacobi polynomials while $X_{A}^{-1}$ and $X_{B}^{-1}$ are constant factors arising from evaluating the Jacobi polynomials at $\tau=0$.

For further details see [15]. The case $l=p$ is special and the central point in the discussion of this section:

Proposition 1. For $p \geq 2, \ell=p,-p \leq m \leq p$ the solution to equations (18a) and 18b) is given by

$$
\begin{gathered}
a_{0, p ; p, m}(\tau)=\left(\frac{1-\tau}{2}\right)^{p+2}\left(\frac{1+\tau}{2}\right)^{p-2}\left(E_{p, m}+E_{p, m}^{*} \int_{0}^{\tau} \frac{d s}{(1+s)^{p-1}(1-s)^{p+3}}\right), \\
a_{4, p ; p, m}(\tau)=\left(\frac{1+\tau}{2}\right)^{p+2}\left(\frac{1-\tau}{2}\right)^{p-2}\left(I_{p, m}+I_{p, m}^{*} \int_{0}^{\tau} \frac{d s}{(1-s)^{p-1}(1+s)^{p+3}}\right) .
\end{gathered}
$$

where $E_{p, l, m}, E_{p, l, m}^{*}$ and $I_{p, l, m}, I_{p, l, m}^{*}$ are integration constants.

Remark 2. Observe that if $E_{p, l, m}^{*}$ and $I_{p, l, m}^{*}$ vanish then the solutions $a_{n, p, \ell, m}$ are polynomial. To understand the effect of non-vanishing constants $E_{p, l, m}^{*}$ and $I_{p, l, m}^{*}$, observe that using partial fractions, the integrals of Proposition 1 give rise to logarithmic terms in the solution. More precisely, one has that

$$
\begin{aligned}
\int_{0}^{\tau} \frac{d s}{(1 \pm s)^{p-1}(1 \mp s)^{p+3}}= & A_{*} \ln (1-\tau)+\frac{A_{p \pm 2}}{(1-\tau)^{p \pm 2}}+\cdots+\frac{A_{1}}{(1-\tau)}+A_{0} \\
& +B_{*} \ln (1+\tau)+\frac{B_{p \mp 2}}{(1+\tau)^{p \mp 2}}+\cdots+\frac{B_{1}}{(1+\tau)}
\end{aligned}
$$

where $A_{i}$ and $B_{i}$ are constants.

The integration constants $E_{p, l, m}^{*}$ and $I_{p, l, m}^{*}$ associated to the logarithmic singularities in the solutions for the $\ell=p$ modes can be related to initial data via the linearisation of the Bach spinor $B_{A B C D}$ - see [26]. This gives rise to the following regularity condition ensuring the vanishing of $E_{p, l, m}^{*}$ and $I_{p, l, m}^{*}$ : 
Proposition 2. The solution to the spin-2 equation on $(\mathcal{M}, \boldsymbol{\eta})$ extends analytically to the critical sets $\mathcal{I}^{ \pm}$if and only if on the initial hypersurface

$$
\mathcal{S}_{\star} \equiv\{p \in \mathcal{M} \mid \tau(p)=0\},
$$

the regularity condition

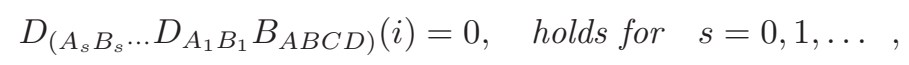

where $B_{A B C D}$ is the linearised Bach spinor, which can be written in terms of the conformal factor and the spin-2 field as

$$
B_{A B C D}=2 D_{E(A} \Omega \phi_{B C D)}^{E}+\Omega D_{E(A} \phi_{B C D)}{ }^{E},
$$

where $D_{A B}$ is the spinorial counterpart of the Levi-Civita connection of $(\boldsymbol{h}, \mathcal{S})$ where $\boldsymbol{h}$ is the pull back of $\boldsymbol{\eta}$ to $\mathcal{S}_{\star}$ and $\left.\Omega \equiv \Theta\right|_{\mathcal{S}_{\star}}=\rho$.

Remark 3. It is important to stress that the regularity condition (21) does not imply, say, that $B_{A B C D}=0$ in a neighbourhood of $i$. This can be seen in the case that $B_{A B C D}$ is analytic in a

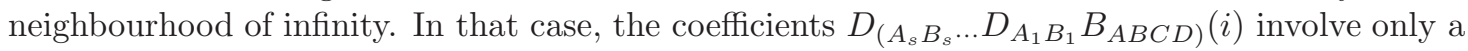
part of the derivatives at order $p$ - the other parts involve a curl and a divergence. For example, if $p=1$ one has that the various components of $D_{A_{1} B_{1}} B_{A B C D}(i)$ are given by

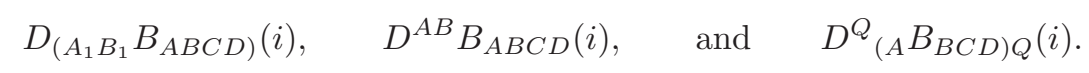

A more detailed discussion of this issue and the relation between the irreducible decomposition of spinors and Taylor expansions can be found in [7, Section 3.3.

Remark 4. The regularity condition (21) is conformally invariant. This follows from the transformation of the linearised Bach spinor and the fact that it is a condition at a point - see e.g. [32, Section 19.3 where the conformal properties of the analogue condition for the (non-linearised) Bach spinor are discussed.

Further discussion of the consequences of logarithmic singularities in the asymptotic expansions of the spin-2 fields at the critical sets and its connection to the (non) peeling properties of $\phi_{A B C D}$ can be found in [26. The non-linear version (for the full conformal Einstein field equations) of the regularity condition (21) was derived in [7. The polyhomogeneous peeling behaviour consistent with the existence of logarithmic singularities in the rescaled Weyl spinor and the Weyl spinor are given in [7, 27, 28, 29] and [14] respectively.

\section{The space-spinor formalism}

In this section we briefly recall the space-spinor formalism and its naturally associated $1+3$ split - see e.g. [21, 32. Although similar in spirit, this formalism is different in some key aspects from the more common $3+1$ split. The most fundamental difference being that the $1+3$ split is adapted to a preferred direction given by a vector field $\tau^{a}$ instead of a foliation. In other words, the vector $\tau^{a}$ is, in general, not hypersurface orthogonal and hence, the distribution generated by $\tau^{a}$ is not integrable. The subsequent analysis will further refinement of a $1+1+2$ spinorial split. This is analogous to the $1+3$ split when an additional direction $\rho^{a}$ is singled out - see [32, 21, 24, 23, 16] for further discussion on the space-spinor formalism, the $1+3$ split, the $1+1+2$ split and applications. The basic elements of this formalism will be discussed on a general manifold with metric $(\mathcal{M}, \boldsymbol{g})$ and towards the end of this section, some results specific for Friedrich's representation of the spatial infinity of the Minkowski spacetime $(\mathcal{M}, \boldsymbol{\eta})$ will be given. 


\subsection{The general formalism}

Let $(\mathcal{M}, \boldsymbol{g})$ be a manifold equipped with a Lorentzian metric and let $\left\{e_{\boldsymbol{a}}{ }^{a}\right\}$ denote an orthonormal frame. That is, one has

$$
g^{a b}=\eta^{a b} e_{\boldsymbol{a}}^{a} e_{\boldsymbol{b}}^{b}
$$

where $\eta^{\boldsymbol{a b}} \equiv \operatorname{diag}(1,-1,-1,-1)$. An associated null frame $\left\{l^{a}, n^{a}, m^{a}, \bar{m}^{a}\right\}$ can be constructed via

$$
l^{a}=\frac{\sqrt{2}}{2}\left(\boldsymbol{e}_{\mathbf{0}}{ }^{a}+\boldsymbol{e}_{\mathbf{3}}{ }^{a}\right), \quad n^{a}=\frac{\sqrt{2}}{2}\left(\boldsymbol{e}_{\mathbf{0}}{ }^{a}-\boldsymbol{e}_{\mathbf{3}}{ }^{a}\right), \quad m^{a}=\frac{\sqrt{2}}{2}\left(\boldsymbol{e}_{\mathbf{1}}{ }^{a}-\mathrm{i} \boldsymbol{e}_{\mathbf{2}}{ }^{a}\right) .
$$

The spinorial counterpart of this null frame is denoted by $\left\{\boldsymbol{e}_{\boldsymbol{A} \boldsymbol{A}^{\prime}}{ }^{a}\right\}$ and satisfies

$$
g^{a b}=\epsilon^{\boldsymbol{A} \boldsymbol{B}} \epsilon^{\boldsymbol{A}^{\prime} \boldsymbol{B}^{\prime}} e_{\boldsymbol{A} \boldsymbol{A}^{\prime}} e^{a} e_{\boldsymbol{B} \boldsymbol{B}^{\prime}}{ }^{b}
$$

Let $\tau^{A A^{\prime}}$ represent the spinor counterpart of the timelike vector $\sqrt{2} e_{\mathbf{0}}{ }^{a}$. Let $\left\{\epsilon_{\boldsymbol{A}}{ }^{A}\right\}$ be a spinor dyad $\epsilon_{\mathbf{0}}{ }^{A}=o^{A}, \epsilon_{\mathbf{1}}{ }^{A}=\iota^{A}$ such that

$$
\tau^{A A^{\prime}}=\epsilon_{\mathbf{0}}^{A} \epsilon_{\mathbf{0}^{\prime}}^{A^{\prime}}+\epsilon_{\mathbf{1}}{ }^{A} \epsilon_{\mathbf{1}^{\prime}}{ }^{A^{\prime}} .
$$

The latter implies that the spinor $\tau^{A A^{\prime}}$ satisfies

$$
\tau_{A A^{\prime}} \tau^{B A^{\prime}}=\epsilon_{A}^{B}
$$

Consequently, the normalisation is fixed so that $\tau^{A A^{\prime}} \tau_{A A^{\prime}}=2$. This normalisation is consistent with the conventions of 6 .

The Hermitian spinor $\tau^{A A^{\prime}}$ induces a notion of Hermitian conjugation as follows: given a spinor $\mu_{A A^{\prime}}$ its Hermitian conjugate $\mu_{A A^{\prime}}^{\dagger}$ is defined as

$$
\mu_{C D}^{\dagger} \equiv \tau_{C}^{A^{\prime}} \tau_{D}{ }^{A} \overline{\mu_{A A^{\prime}}}=\tau_{C}^{A^{\prime}} \tau_{D}{ }^{A} \bar{\mu}_{A^{\prime} A},
$$

where the bar denotes complex conjugation. This definition is extended to higher valence spinors by requiring that $(\boldsymbol{\pi} \boldsymbol{\mu})^{\dagger}=\boldsymbol{\pi}^{\dagger} \boldsymbol{\mu}^{\dagger}$.

\subsubsection{The space-spinor split}

The Hermitian spinor $\tau^{A A^{\prime}}$ induces a space-spinor split as follows: given a spinor $v_{A A^{\prime}}$ its spacespinor decomposition is determined by

$$
v_{A A^{\prime}}=\frac{1}{2} \tau_{A A^{\prime}} v-\tau_{A^{\prime}} v_{(Q A)},
$$

where $v \equiv v_{Q Q^{\prime}} \tau^{Q Q^{\prime}}$ and $v_{A B} \equiv \tau_{B}{ }^{A^{\prime}} v_{A A^{\prime}}$. Similar decompositions apply to higher valence spinors. The space-spinor split of a spinor $\boldsymbol{\mu}$ with $n$ unprimed indices and $m$ primed indices $\mu_{A_{1}, . . A_{n}, A^{\prime}, \ldots, A_{m}^{\prime}}$ can be succinctly described as the process of transvecting it with $\tau_{B_{1}} A_{1}^{\prime} \cdots \tau_{B_{m}} A_{m}^{\prime}$ to produce its space-spinor counterpart $\mu_{A_{1}, \ldots A_{n}, B_{1}, \ldots B_{m}}$, which in turn is (irreducibly) decomposed in terms of totally symmetric spinors of equal or lower valence - see 32 for a comprehensive discussion.

\subsubsection{The space-spinor decomposition of the connection}

Any general connection $\breve{\nabla}$ —not necessarily the Levi-Civita for which the symbol $\boldsymbol{\nabla}$ is reservedcan be split as

$$
\breve{\nabla}_{A A^{\prime}}=\frac{1}{2} \tau_{A A^{\prime}} \breve{\mathcal{D}}-\tau_{A^{\prime}}{ }^{Q} \breve{\mathcal{D}}_{A Q}
$$

where

$$
\breve{\mathcal{D}} \equiv \tau^{A A^{\prime}} \breve{\nabla}_{A A^{\prime}} \quad \text { and } \quad \breve{\mathcal{D}}_{A B} \equiv \tau_{(B}{ }^{A^{\prime}} \breve{\nabla}_{A) A^{\prime}}
$$


denote, respectively, the Fermi covariant derivative in the direction of $\tau^{A A^{\prime}}$ and the Sen connection of $\breve{\nabla}$ relative to $\tau^{A A^{\prime}}$. It is worth mentioning that even in the case of a Levi-Civita connection $\boldsymbol{\nabla}$, the associated Sen connection $\mathcal{D}$ will have a non-vanishing torsion which is encoded in the derivatives of $\tau^{a}$. In fact, as briefly described in the following, these derivatives play an important role in the space-spinor decomposition of the components of the connection.

In the following, it is convenient to define

$$
\mathcal{X}_{A B C D} \equiv \frac{1}{\sqrt{2}} \tau_{D}{ }^{Q^{\prime}} \tau_{B}{ }^{A^{\prime}} \nabla_{A A^{\prime}} \tau_{C C^{\prime}}
$$

This spinor, encoding the derivatives of $\tau^{a}$, can be decomposed in terms of the reduced spinors

$$
\chi_{A B} \equiv \frac{1}{\sqrt{2}} \tau_{B}{ }^{A^{\prime}} \mathcal{D} \tau_{A A^{\prime}}, \quad \chi_{A B C D} \equiv \frac{1}{\sqrt{2}} \tau_{D}{ }^{C^{\prime}} \mathcal{D}_{A B} \tau_{C C^{\prime}}
$$

The acceleration of $\tau^{a}$ is encoded in $\chi_{A B}$ while $\chi_{A B C D}$ is the Weingarten spinor associated to $\tau^{a}$. If $\mathcal{X}^{Q}{ }_{(A B) Q}=0$ then $\tau^{a}$ is hypersurface orthogonal and the Levi-Civita connection induced on the leaves of the associated foliation, to be denoted by $D_{A B}$, is related to the Sen derivative by

$$
D_{A B} \mu_{C}=\mathcal{D}_{A B} \mu_{C}+\frac{1}{\sqrt{2}} \chi_{A B C}{ }^{Q} \mu_{Q}
$$

Let $\Gamma_{\boldsymbol{A} \boldsymbol{A}^{\prime} \boldsymbol{C} \boldsymbol{D}}$ denote the (spin) connection coefficients associated to the Levi-Civita connection $\boldsymbol{\nabla}$ in the spin dyad $\left\{\epsilon_{\boldsymbol{A}}{ }^{A}\right\}$. Defining $\Gamma_{\boldsymbol{A} \boldsymbol{B} \boldsymbol{C} \boldsymbol{D}} \equiv \tau_{\boldsymbol{B}}{ }^{\boldsymbol{A}^{\prime}} \Gamma_{\boldsymbol{A} \boldsymbol{A}^{\prime} \boldsymbol{C} \boldsymbol{D}}$ and exploiting the Hermitian conjugation operation, it can be shown that the spinor $\mathcal{X}_{A B C D}$ encodes the real part of the connection. More precisely, one has that

$$
\mathcal{X}_{A B C D}=-\frac{1}{\sqrt{2}}\left(\Gamma_{A B C D}+\Gamma_{A B C D}^{\dagger}\right) .
$$

The spinor $\xi_{A B C D}$ encoding the imaginary part of the connection is given by

$$
\xi_{A B C D}=\frac{1}{\sqrt{2}}\left(\Gamma_{A B C D}-\Gamma_{A B C D}^{\dagger}\right) .
$$

\subsubsection{The $1+1+2$ spinor decomposition}

Spinorial $1+1+2$ splits naturally arise in settings where a spacelike vector $\rho^{a}$ is distinguished. Let $\rho^{A A^{\prime}}$ denote the spinorial counterpart of $\rho^{a}$. In the following it is assumed that $\rho^{A A^{\prime}}$ is orthogonal to $\tau^{A A^{\prime}}$ - that is,

$$
\tau_{A A^{\prime}} \rho^{A A^{\prime}}=0
$$

In analogy to equation (24) one can further specialise the dyad $\left\{\epsilon_{A}{ }^{A}\right\}$ so that

$$
\rho^{A A^{\prime}}=\epsilon_{\mathbf{0}}{ }^{A} \epsilon_{\mathbf{0}^{\prime}}{ }^{A^{\prime}}-\epsilon_{\mathbf{1}}^{A} \epsilon_{\mathbf{1}^{\prime}}{ }^{A^{\prime}} .
$$

This choice of dyad is consistent with equation (24), but reduces the freedom available in the dyad to $U(1)$ transformations. As a consequence of equation (29), the spinor $\rho^{A A^{\prime}}$ satisfies

$$
\rho_{A A^{\prime}} \rho^{B A^{\prime}}=-\delta_{A}^{B}
$$

The latter implies $\rho_{A A^{\prime}} \rho^{A A^{\prime}}=-2$ consistent with the assumption that $\rho^{a}$ is a spacelike vector. Consistent with the previous discussion we identify $\rho^{a}$ with $\sqrt{2} e_{\mathbf{3}}{ }^{a}$. Additionally, the orthogonality condition (28), implies that $\rho_{A B}=\rho_{(A B)}$ where

$$
\rho_{A B} \equiv \tau_{B}{ }^{A^{\prime}} \rho_{A A^{\prime}} .
$$


Further discussion on the spinorial $1+1+2$ split can be found in [24, 23, 16].

Particularising the previous general discussion to Friedrich's representation of the spatial infinity of the Minkowski spacetime and using the lift to the fibre space of the null frame (9a)(9d) and exploiting (23) one can directly read

$$
\boldsymbol{\tau}=\sqrt{2} \boldsymbol{\partial}_{\tau}, \quad \boldsymbol{\rho}=\sqrt{2}\left(\rho \boldsymbol{\partial}_{\rho}-\tau \boldsymbol{\partial}_{\tau}\right) .
$$

Using the latter expressions and the frame a calculation carried out using the suite xAct for tensor and spinorial manipulations in Mathematica - see [13 — gives that

$$
\chi_{A B C D}=0, \quad \chi_{A B}=-\frac{2}{\sqrt{2}} \rho_{A B} .
$$

The expressions for the spin connection coefficients $\Gamma_{\boldsymbol{A} \boldsymbol{A}^{\prime}}{ }^{B}{ }_{C}$ in the extended bundle space framework were given in Section 2.2

\section{The staticity condition}

In this section we formulate a suitable staticity condition for spin-2 fields in the Minkowski spacetime. We then proceed to express this condition in terms of the corresponding rescaled fields in the conformal representation of the Minkowski spacetime discussed in Section 2. The latter will allow in turn to relate the staticity condition with the regularity condition discussed in Section 3

\subsection{Staticity conditions in the physical spacetime}

Let $\tilde{\phi}_{a b c d}$ be a spin-2 field on Minkowski spacetime $\left(\mathbb{R}^{4}, \tilde{\boldsymbol{\eta}}\right)$. In other words, let $\tilde{\phi}_{a b c d}$ be a tensor satisfying

$$
\tilde{\nabla}^{a} \tilde{\phi}_{a b c d}=0 .
$$

where $\tilde{\phi}_{a b c d}$ possesses the same symmetries of the Weyl tensor. Its spinorial counterpart can be decomposed as

$$
\tilde{\phi}_{A A^{\prime} B B^{\prime} C C^{\prime} D D^{\prime}}=\tilde{\phi}_{A B C D} \tilde{\epsilon}_{A^{\prime} B^{\prime}} \tilde{\epsilon}_{C^{\prime} D^{\prime}}+\overline{\tilde{\phi}}_{A^{\prime} B^{\prime} C^{\prime} D^{\prime}} \tilde{\epsilon}_{A B} \tilde{\epsilon}_{C D},
$$

where $\tilde{\phi}_{A B C D}=\tilde{\phi}_{(A B C D)}$. The spinor field $\tilde{\phi}_{A B C D}$ satisfies

$$
\tilde{\nabla}_{A^{\prime}}{ }^{A} \tilde{\phi}_{A B C D}=0 .
$$

\subsubsection{Definition}

Consistent with the notation of Section 2 let $\tilde{x}^{\mu}=\left(\tilde{t}, \tilde{x}^{i}\right)$ denote Cartesian coordinates. In the following we make use of the following ad hoc notion of staticity for the spin-2 field:

Definition 1. The spin-2 field $\tilde{\phi}_{\text {abcd }}$ on the Minkowski spacetime is static if and only if

$$
\mathcal{L}_{\tilde{\xi}} \tilde{\phi}_{a b c d}=0, \quad \text { with } \quad \tilde{\xi}=\partial_{\tilde{t}} .
$$

In view of the latter definition it is convenient to introduce the zero-quantity

$$
\tilde{Z}_{a b c d} \equiv \mathcal{L}_{\tilde{\xi}} \tilde{\phi}_{a b c d}, \quad \text { with } \quad \tilde{\xi}=\boldsymbol{\partial}_{\tilde{t}} .
$$

Expressing the Lie derivative in terms of covariant derivatives and using the fact that the Killing vector $\partial_{\tilde{t}}$ in the Minkowski spacetime is covariantly constant so that $\tilde{\nabla}_{a} \tilde{\xi}^{e}=0$, one concludes that

$$
\tilde{Z}_{a b c d}=\tilde{\xi}^{e} \tilde{\nabla}_{e} \tilde{\phi}_{a b c d}
$$




\subsubsection{Propagation equations}

The next step in our analysis is to formulate the spacetime staticity notion encoded in Definition 1 in terms of conditions on the initial data for the spin-2 equation.

Using equation (32) and exploiting the fact that

$$
\left[\mathcal{L}_{\tilde{\xi}}, \tilde{\nabla}\right] \boldsymbol{T}=0
$$

for any Killing vector $\tilde{\boldsymbol{\xi}}$ and any tensor field $\boldsymbol{T}$ — see [34 — one concludes that

$$
\tilde{\nabla}^{a} \tilde{Z}_{a b c d}=0 \text {. }
$$

It can be readily verified that the spinorial version of equation (36) is

$$
\tilde{\nabla}_{A^{\prime}}{ }^{A} \tilde{Z}_{A B C D}=0,
$$

where $\tilde{Z}_{A A^{\prime} B B^{\prime} C C^{\prime} D D^{\prime}}$ is the spinorial counterpart of $\tilde{Z}_{a b c d}$ and is related to $Z_{A B C D}$ via

$$
\tilde{Z}_{A A^{\prime} B B^{\prime} C C^{\prime} D D^{\prime}}=\tilde{Z}_{A B C D} \tilde{\epsilon}_{A^{\prime} B^{\prime}} \tilde{\epsilon}_{C^{\prime} D^{\prime}}+\overline{\tilde{Z}}_{A^{\prime} B^{\prime} C^{\prime} D^{\prime}} \tilde{\epsilon}_{A B} \tilde{\epsilon}_{C D} .
$$

In order to extract the content of equation (37) we make use of the space-spinor formalism. Setting $\tilde{\tau}^{A A^{\prime}}=\sqrt{2} \tilde{\xi}^{A A^{\prime}}$ where $\tilde{\xi}^{A A^{\prime}}$ denotes the spinorial counterpart of $\tilde{\xi}^{a}$, a direct calculation using the space-spinor formalism described in Section 4 shows that equation (37) can be split as

$$
\begin{aligned}
& \tilde{\mathcal{D}} \tilde{Z}_{A B C D}-2 \tilde{\mathcal{D}}^{Q}{ }_{(A} \tilde{Z}_{B C D) Q}=0, \\
& \tilde{\mathcal{D}}^{A B} \tilde{Z}_{A B C D}=0 .
\end{aligned}
$$

with $\tilde{\mathcal{D}}$ and $\tilde{\mathcal{D}}_{A B}$ as defined in Section 4 The evolution equations (39a) imply a symmetric hyperbolic system for the components of $\tilde{Z}_{A B C D}$. This observation follows by formal analogy to the analysis of the hyperbolic reductions of the spin-2 equation - see 32 for further discussion on the hyperbolicity analysis of this type of spinorial equations. Consequently, exploiting the standard uniqueness result for symmetric hyperbolic systems, one has that

$$
\left.\tilde{Z}_{A B C D}\right|_{\tilde{\mathcal{S}}}=0 \quad \Longleftrightarrow \quad \tilde{Z}_{A B C D}=0 \quad \text { in } \quad D^{+}(\tilde{\mathcal{S}}),
$$

with $\tilde{\mathcal{S}}$, say, a Cauchy hypersurface of the Minkowski spacetime.

In summary, it follows from the previous discussion that the requirement of staticity for the spin-2 field on the Minkowski spacetime (as given by Definition 1 ) is equivalent to the initial data condition

$$
\left.\tilde{Z}_{A B C D}\right|_{\tilde{\mathcal{S}}}=0 .
$$

In the following we refer to (41) as to the staticity condition. Condition (41) will impose some restrictions on the initial data for the physical spin-2 field $\tilde{\phi}_{A B C D}$. In the following we analyse the relation between this condition and the regularity condition of Proposition 2 In order to do so, the two conditions needs to be written in the same framework.

\subsection{Staticity condition in the unphysical spacetime}

In this subsection we recall general conformation formulate. These expressions are then particularised to the case of the Minkowski spacetime $\left(\mathbb{R}^{4}, \tilde{\boldsymbol{\eta}}\right)$ and the conformal extension $(\mathcal{M}, \boldsymbol{\eta})$ discussed in Section 2 Finally, the staticity condition (41) is recast in terms of the (unphysical) spin-2 field $\phi_{A B C D}$ propagating in $(\mathcal{M}, \boldsymbol{\eta})$. 


\subsubsection{Conformal transformation formulae}

We start by recalling that:

Lemma 2. Let $(\mathcal{M}, \boldsymbol{g})$ and $(\tilde{\mathcal{M}}, \tilde{\boldsymbol{g}})$ two Lorentzian manifolds equipped metrics related through a conformal transformation

$$
\boldsymbol{g}=\Xi^{2} \tilde{\boldsymbol{g}} .
$$

Let $\tilde{\phi}_{a b c d}$ be a tensor with the same symmetries of the Weyl tensor then,

$$
\tilde{\nabla}^{a} \tilde{\phi}_{a b c d}=0 \Longrightarrow \nabla^{a} \phi_{a b c d}=0
$$

where $\tilde{\phi}_{a b c d}=\Xi^{-1} \phi_{a b c d}$. In terms of their reduced spinorial counterparts $\tilde{\phi}_{A B C D}$ and $\phi_{A B C D}$ one similarly has that

$$
\tilde{\nabla}_{A^{\prime}}{ }^{A} \tilde{\phi}_{A B C D}=0 \Longrightarrow \nabla_{A^{\prime}}{ }^{A} \phi_{A B C D}=0
$$

where $\phi_{A B C D}=\Xi^{-1} \tilde{\phi}_{A B C D}$.

This classical result follows from a direct calculation using the conformal transformation formulae relating the connections $\boldsymbol{\nabla}$ and $\tilde{\nabla}$ - see [22, 20]. We also note the following:

Lemma 3. If $(\tilde{\mathcal{M}}, \tilde{\boldsymbol{g}})$ admits a Killing vector $\tilde{\boldsymbol{\xi}}$, namely, $\tilde{\nabla}_{(a} \tilde{\xi}_{b)}=0$, then $\tilde{\boldsymbol{\xi}}$ gives rise to a conformal Killing vector $\boldsymbol{\xi}$ on $(\mathcal{M}, \boldsymbol{g})$. That is, one has

$$
\nabla_{(a} \xi_{b)}-\frac{1}{4} g_{a b} \nabla_{c} \xi^{c}=0, \quad \text { where } \quad \nabla_{a} \xi^{a}=4 \Xi \xi^{a} \nabla_{a} \Xi
$$

with

$$
\xi^{a}=\tilde{\xi}^{a}, \quad \xi_{a}=\Xi^{-2} \tilde{\xi}_{a} .
$$

This result is straightforwardly obtained by direct substitution — see also [18.

Remark 5. The conformal factor appears in the second expression in equation (46) because the indices of tensors in $(\tilde{\mathcal{M}}, \tilde{\boldsymbol{g}})$ are, by definition, raised and lowered using $\tilde{\boldsymbol{g}}$ while for tensors in $(\mathcal{M}, \boldsymbol{g})$, the metric $\boldsymbol{g}$ is used. For the case of the spin-2 equation it is enough to identify explicitly the conformal Killing vector on $(\mathcal{M}, \boldsymbol{\eta})$ associated to the timelike Killing vector in $\left(\mathbb{R}^{4}, \tilde{\boldsymbol{\eta}}\right)$. However for more general spacetimes one would need to make use of the conformal Killing initial data equations of [18].

\subsubsection{Propagation equations in the unphysical spacetime}

Most of the subsequent discussion can be made general without having to make use of the particular features of the conformal extension $(\mathcal{M}, \boldsymbol{\eta})$ of the Minkowski spacetime.

As before, let $\tilde{Z}_{a b c d} \equiv \mathcal{L}_{\xi} \tilde{\phi}_{a b c d}$. Using that $\tilde{\phi}_{a b c d}=\Xi^{-1} \phi_{a b c d}$ one has that

$$
\tilde{Z}_{a b c d}=\phi_{a b c d} \mathcal{L}_{\xi} \Xi+\Xi \mathcal{L}_{\xi} \phi_{a b c d} .
$$

A direct spinorial translation of the latter equation gives

$$
\tilde{Z}_{A A^{\prime} B B^{\prime} C C^{\prime} D D^{\prime}}=\phi_{A A^{\prime} B B^{\prime} C C^{\prime} D D^{\prime}} \mathcal{L}_{\xi} \Xi+\Xi \mathcal{L}_{\xi} \phi_{A A^{\prime} B B^{\prime} C C^{\prime} D D^{\prime}}
$$

Remark 6. It is worth making a word of caution regarding the notion of Lie derivative for spinorial fields. Give a spinor $\mu^{A}$, its Lie derivative $\mathcal{L}_{\xi} \mu^{A}$ is only well defined when $\xi^{a}$ is a conformal Killing vector - see [20]. This is not a problem in the current set up as $\xi^{a}$ is assumed from the outset to be a conformal Killing vector. Moreover, the conformal Killing vector equation can be expressed in spinorial terms as

$$
\mathcal{L}_{\xi} \epsilon^{A B}=\lambda \epsilon^{A B}
$$


where

$$
\nabla_{A A^{\prime}} \xi^{A A^{\prime}}=-2(\lambda+\bar{\lambda}) .
$$

With this notation at hand the Lie-derivative along $\xi^{a}$ of a valence- 1 spinor $\mu^{A}$ is given by

$$
\mathcal{L}_{\xi} \mu^{A}=\xi^{Q Q^{\prime}} \nabla_{Q Q^{\prime}} \mu^{A}-h_{Q}{ }^{A} \mu^{Q},
$$

where

$$
h_{A}{ }^{B}=\frac{1}{2}\left(\epsilon_{A}^{B} \bar{\lambda}+\nabla_{A Q^{\prime}} \xi^{B Q^{\prime}}\right),
$$

- see 20] for further details. As discussed in 20 the divergence of the conformal Killing vector only fixes the real part of $\lambda$ and a geometrically natural choice is to set $\lambda=\bar{\lambda}$-consistent with a conformal rescaling using a real function $\Xi$ instead of a complex one.

Applying $\mathcal{L}_{\xi}$ to the irreducible decomposition for the spinorial counterpart of $\phi_{a b c d}$ - the analogue of equation (33) - gives

$$
\mathcal{L}_{\xi} \phi_{A A^{\prime} B B^{\prime} C C^{\prime} D D^{\prime}}=\epsilon_{A^{\prime} B^{\prime}} \epsilon_{C^{\prime} D^{\prime}} \mathcal{L}_{\xi} \phi_{A B C D}-2 \bar{\lambda} \phi_{A B C D} \epsilon_{A^{\prime} B^{\prime}} \epsilon_{C^{\prime} D^{\prime}}+\text { c.c. },
$$

where c.c. denotes the complex conjugate of the displayed expression. Now, defining

$$
Z_{A B C D} \equiv \Xi^{-2} \tilde{Z}_{A B C D}
$$

and observing that $\epsilon_{A B}=\Xi \tilde{\epsilon}_{A B}$, consistent with the conformal transformation (42), gives

$$
\tilde{Z}_{A A^{\prime} B B^{\prime} C C^{\prime} D D^{\prime}}=Z_{A B C D} \epsilon_{A^{\prime} B^{\prime}} \epsilon_{C^{\prime} D^{\prime}}+\bar{Z}_{A^{\prime} B^{\prime} C^{\prime} D^{\prime}} \epsilon_{A B} \epsilon_{C D} .
$$

Substituting equations (52) and (53) into equation (47) and symmetrising gives

$$
Z_{A B C D}=\Xi \mathcal{L}_{\xi} \phi_{A B C D}-2 \Xi \bar{\lambda} \phi_{A B C D}+\phi_{A B C D} \mathcal{L}_{\xi} \Xi .
$$

Unwrapping the expression for the Lie derivative $\mathcal{L}_{\xi} \phi_{A B C D}$, using the generalisation of equation (50) for a totally symmetric valance-4 spinor (see [20]) leads to

$$
\mathcal{L}_{\xi} \phi_{A B C D}=\xi^{Q Q^{\prime}} \nabla_{Q Q^{\prime}} \phi_{A B C D}+2 \phi_{Q(A B C} \nabla_{D) Q^{\prime}} \xi^{Q Q^{\prime}}+2 \bar{\lambda} \phi_{A B C D} .
$$

Accordingly, equation (54) can be rewritten in a more explicit form as

$$
Z_{A B C D}=\Xi \xi^{Q Q^{\prime}} \nabla_{Q Q^{\prime}} \phi_{A B C D}+\phi_{A B C D} \mathcal{L}_{\xi} \Xi+2 \phi_{Q(A B C} \nabla_{D) Q^{\prime}} \xi^{Q Q^{\prime}} .
$$

Notice that the term $\bar{\lambda}$ present in equation (55) is absent once one expands the Lie derivative of $\phi_{A B C D}$.

\subsubsection{Space-spinor decomposition of the staticity condition}

In view of the eventual evaluation of $Z_{A B C D}$ on the initial hypersurface $\mathcal{S}$, a $1+3$ split of equation (56) is in order. Let $\tau^{A A^{\prime}}$ correspond to the vector $\sqrt{2} \boldsymbol{\partial}_{\tau}$ with $\boldsymbol{g}\left(\boldsymbol{\partial}_{\tau}, \boldsymbol{\partial}_{\tau}\right)=1$. To employ the space-spinor split induced by $\tau^{A A^{\prime}}$, define

$$
\xi \equiv \frac{1}{\sqrt{2}} \xi^{A A^{\prime}} \tau_{A A^{\prime}}, \quad \xi_{A B} \equiv \xi_{A^{\prime}(A} \tau_{B)} A^{\prime},
$$

so that $\xi_{A A^{\prime}}$ can be decomposed as

$$
\xi_{A A^{\prime}}=\frac{1}{\sqrt{2}} \xi \tau_{A A^{\prime}}+\xi_{A}^{B} \tau_{B A^{\prime}} .
$$


The decomposition (58) and the space spinor formalism can be employed to rewrite each of the terms in the right hand side of equation (56) as follows: for the first term a short calculation gives

$$
\xi^{F A^{\prime}} \nabla_{F A^{\prime}} \phi_{A B C D}=\frac{1}{\sqrt{2}} \xi \mathcal{D} \phi_{A B C D}+\xi^{F G} \mathcal{D}_{G F} \phi_{A B C D},
$$

and for the second term

$$
\mathcal{L}_{\xi} \Xi=\frac{1}{\sqrt{2}} \xi \mathcal{D} \Xi+\xi^{A B} \mathcal{D}_{A B} \Xi
$$

For the last term in the righthand side of equation (56) a longer calculation shows that

$$
\begin{array}{r}
4 \phi_{B C D F}{ }^{F} \nabla_{A A^{\prime}} \xi_{F}{ }^{A^{\prime}}=\chi_{A}{ }^{F}\left(2 \xi_{F}{ }^{G} \phi_{B C D G}-\sqrt{2} \phi_{B C D F} \xi\right)-2 \phi_{B C D F} \mathcal{D} \xi_{A}{ }^{F}+\sqrt{2} \phi_{A B C D} \mathcal{D} \xi \\
-4 \phi_{B C D G}\left(\xi \chi_{A}{ }^{F}{ }_{F}{ }^{G}+\sqrt{2} \xi^{F G} \chi_{A}{ }^{H}{ }_{F H}-\mathcal{D}_{A F} \xi^{F G}\right)+2 \sqrt{2} \phi_{B C D F} \mathcal{D}_{A}{ }^{F} \xi
\end{array}
$$

where $\chi_{A B C D}$ and $\chi_{A B}$ denote encode the tangential and normal derivatives of $\tau^{a}$ as defined in Section 4

Remark 7. Observe that expressions (59a)- (59c) contain both normal and tangential derivatives of $\xi, \xi_{A B}$ and $\phi_{A B C D}$. The normal derivatives can be replaced by tangential derivatives exploiting the conformal Killing vector and the spin-2 equations satisfied by $\xi_{A A^{\prime}}$ and $\phi_{A B C D}$, respectively. More precisely, one has that:

(a) The direct spinorial translation of the conformal Killing vector equation (45) reads

$$
\nabla_{A A^{\prime}} \xi_{B B^{\prime}}+\nabla_{B B^{\prime}} \xi_{A A^{\prime}}-\frac{1}{2} \epsilon_{A B} \epsilon_{A^{\prime} B^{\prime}} \nabla_{C C^{\prime}} \xi^{C C^{\prime}}=0
$$

Substituting the decomposition (58) into equation (60), using the space-spinor split and solving for the normal derivatives of $\xi$ and $\xi_{A B}$ one obtains

$$
\begin{aligned}
& \mathcal{D} \xi=\Xi \xi \mathcal{D} \Xi+\frac{1}{\sqrt{2}} \chi^{A B} \xi_{A B}+\frac{2}{\sqrt{2}} \xi^{A B} \mathcal{D}_{A B} \Xi \\
& \mathcal{D} \xi_{A P}=\frac{1}{2} \chi_{P}{ }^{B} \xi_{A B}+\frac{1}{2} \chi_{A}{ }^{B} \xi_{P B}-\frac{1}{\sqrt{2}} \chi_{A P} \xi+\sqrt{2} \xi^{B C} \chi_{A P B C}-\sqrt{2} \mathcal{D}_{A P} \xi
\end{aligned}
$$

(b) Similarly, from the spin-2 equation (44), using the space-spinor formalism and solving for the normal derivatives of $\phi_{A B C D}$ one finds that

$$
\mathcal{P} \phi_{A B C D}=2 \mathcal{D}^{Q}{ }_{(A} \phi_{B C D) Q}
$$

Using equations (61a)- (61b) to replace the normal derivatives in (59) and substituting this result into equation (56) one obtains an expression which only contains tangential (Sen) derivatives. This long expression is not very insightful.

\subsubsection{Specific expressions for the Minkowski spacetime}

At this point it is convenient to particularise the discussion for the case of the conformal extension $(\mathcal{M}, \boldsymbol{\eta})$ of the Minkowski spacetime.

The most important simplification that occurs in the present case, is that the conformal Killing vector $\boldsymbol{\xi}$ associated to $\tilde{\boldsymbol{\xi}}=\boldsymbol{\partial}_{\tilde{t}}$ is, in fact, a Killing vector of the unphysical spacetime $(\mathcal{M}, \boldsymbol{\eta})$. To see this, notice that using the coordinate transformations (6) and $t=\tau \rho$, the conformal factor $\Theta$ given in equation (10) when expressed in the physical coordinates $(\tilde{t}, \tilde{\rho})$ simply reads

$$
\Theta=-\frac{1}{\tilde{\rho}} .
$$


Observing that $\boldsymbol{\xi}=\tilde{\boldsymbol{\xi}}=\boldsymbol{\partial}_{\tilde{t}}$, using equation (62) and the second expression in equation (45) one concludes that $\boldsymbol{\xi}=\boldsymbol{\partial}_{\tilde{t}}$ is a Killing vector in $(\mathcal{M}, \boldsymbol{\eta})$. Moreover, a direct application of the chain rule using equations (6) and $t=\tau \rho$ gives

$$
\boldsymbol{\xi}=\boldsymbol{\partial}_{\tilde{t}}=\Theta \boldsymbol{\partial}_{\tau}+2 \rho^{2} \tau \boldsymbol{\partial}_{\rho} .
$$

Using the equation (63) one can identify the Killing shift and lapse $\xi$ and $\xi_{A B}$. A calculation observing (30) gives

$$
\xi=2 \rho \tau^{2}+\Theta, \quad \xi^{A B}=\sqrt{2} \rho \tau \rho^{A B} .
$$

\subsubsection{The final condition}

Observing that $\mathcal{S} \backslash i^{0}$ and $\tilde{\mathcal{S}}$ are diffeomorphic and using that $Z_{A B C D}=\Theta^{2} \tilde{Z}_{A B C D}$ the staticity condition (41) can be expressed as

$$
\left.\tilde{Z}_{A B C D}\right|_{\tilde{\mathcal{S}}}=\left.\Theta^{2} Z_{A B C D}\right|_{\mathcal{S} \backslash i^{0}}=0 .
$$

Thus, by continuity it follows, in fact that

$$
\left.Z_{A B C D}\right|_{\mathcal{S}}=0 .
$$

Using this formulation of the staticity condition and substituting equations (31), (64) along with (61a) - (61b) and (59) into equation (56) one finds, after a long computation that the staticity condition can be rewritten in the very compact form

$$
\left.Z_{A B C D}\right|_{\mathcal{S}}=\left.\sqrt{2} \mathcal{D}_{(A}^{Q}\left(\rho^{2} \phi_{B C D) Q}\right)\right|_{\mathcal{S}}=0
$$

Although in the present case $\tau^{a}$ is not hypersurface orthogonal, one nevertheless has that (27) holds on $\mathcal{S}$. Hence, considering the pull back of equations (31) and (27) to $\mathcal{S}$ one has

$$
\left.Z_{A B C D}\right|_{\mathcal{S}}=\left.\sqrt{2} D_{(A}{ }^{Q}\left(\rho^{2} \phi_{B C D) Q}\right)\right|_{\mathcal{S}}
$$

Now, recalling that the linearisation of the Bach tensor is given in terms of $\phi_{A B C D}$ and $\Omega \equiv \Theta_{\mathcal{S}}$ by

$$
B_{A B C D}=D_{(A}{ }^{Q}\left(\Omega^{2} \phi_{B C D) Q}\right) .
$$

Hence, using (66) and (67) and the above observations one concludes that

$$
Z_{A B C D}=\sqrt{2} B_{A B C D} .
$$

Remark 8. It can be verified that the linearised Bach spinor $B_{A B C D}$ transforms homogeneously under conformal transformations. More precisely, under the rescaling $\boldsymbol{h} \mapsto \phi^{2} \boldsymbol{h}$ one has that

$$
B_{A B C D}^{\prime} \mapsto \phi^{-1} B_{A B C D} .
$$

It follows that the condition $B_{A B C D}=0$ on $\mathcal{S}$ is conformally invariant under rescalings of the initial metric.

The discussion of this section can be summarised in the following:

Theorem 1. A necessary and sufficient condition for a spin-2 field $\phi_{A B C D}$ over $(\mathcal{M}, \boldsymbol{\eta})$ to be static in the sense of Definition 1 is that it satisfies the conformally invariant condition

$$
B_{A B C D}=0 \text { on } \mathcal{S} \text {. }
$$


It follows from the above result that any static spin-2 field trivially satisfies the regularity condition of (21). Accordingly, one has the following:

Corollary 1. Static initial data for the spin-2 field gives rise to a solution $\phi_{A B C D}$ that extends analytically to the the critical sets $\mathcal{I}^{ \pm}$. In particular, the solution is smooth at $\mathscr{I}^{ \pm}$.

Moreover, consistent with Remark 3, we have the following main conclusion of the present analysis:

Corollary 2. Initial data satisfying the regularity condition (21) does not correspond, in general, to static initial data for the spin-2 field in a neighbourhood of $i$.

\section{Conclusions}

The objective of this article has been to analyse the relation between a regularity condition on initial data for the spin-2 field which ensures that the associated solutions extend smoothly (and in fact, analytically) through the critical sets $\mathscr{I}^{+}$where null infinity meets spatial infinity. Making use of the estimates for the solutions to the spin-2 equation developed in 8 ] it follows that regularity at the critical sets implies smoothness at null infinity, $\mathscr{I}^{ \pm}$. In contrast to the case of the Einstein field equations (the ultimate motivation of our analysis) where smoothness at null infinity is closely related to the staticity of the initial data (see e.g. 10, 11, 33, 31), for the linear spin-2 field there exists, a priori, large classes of data satisfying the regularity condition on the linearised Bach spinor which are not static. The underlying reason for this difference can be understood in terms of the more stringent set of (nonlinear) constraints that the initial data sets for the Einstein field equations have to satisfy. Observe also, that while the staticity condition introduced in Definition 1 leads to a linear condition on the initial data, the conformal static equations (see e.g. 32, Chapter 19) are a nonlinear system.

The results of our analysis thus, serve as a cautionary note towards inferring asymptotic properties of solutions to the Einstein field equations from linearised models. One cannot expect to be able to capture the whole richness of the Einstein field equations through the analysis of linearised field equations.

Finally, we observe that a similar analysis can, in principle, be carried out for other massless field equations (e.g. the Dirac and Maxwell fields). Of more interest for the Einstein field equations is the analysis of nonlinear systems like the Maxwell-Dirac or Maxwell-scalar field systems — see e.g. [17].

\section{Acknowledgements}

The problem addressed in this article was motivated by a conversation with Helmut Friedrich at the Erwin Schrödinger Institute (ESI) during the Workshop: Geometric Transport Equations in General Relativity in 2017. We have also profited from discussions with the members of the online Conformal/spinorial workshop held weekly during the months of lockdown in 2020/2021: Alfonso Garcia-Parrado, David Hilditch, Alex Vañó-Viñuales, Marica Minucci, Mariem Magdy Ali Mohamed, Greg Taujanskas, Peng Zhao, Tongtong Hu and Marco Luna. During part of this project, EG was supported via the European Union (through the PO FEDER-FSE Bourgogne 2014/2020 program) and the EIPHI Graduate School (contract ANR-17-EURE-0002) as part of the ISA 2019 project. EG also acknowledges support by the FCT (Portugal) 2020.03845.CEECIND during the last stages of this work. 


\section{References}

[1] M. M. Ali Mohammed \& J. A. Valiente Kroon, A comparison of Ashtekar's and Friedrich's formalisms of spatial infinity, in arXiv:2103.02389[gr-qc], to appear in Class. Quantum Grav. (2021).

[2] R. Beig, Conformal properties of static spacetimes, Class. Quantum Grav. 8, 263 (1991).

[3] P. T. Chruściel \& E. Delay, On mapping properties of the general relativistic constraint operator in weighted function spaces, with applications, Mem. Soc. Math. France 94, 1 (2003).

[4] J. Corvino \& R. Schoen, On the asymptotics for the Einstein Constraint Vacuum Equations, J. Diff. Geom. 73, 185 (2006).

[5] H. Friedrich, On static and radiative space-times, Comm. Math. Phys. 119, 51 (1988).

[6] H. Friedrich, On the global existence and the asymptotic behaviour of solutions to the Einstein-Maxwell-Yang-Mills equations, J. Diff. Geom. 34, 275 (1991).

[7] H. Friedrich, Gravitational fields near space-like and null infinity, J. Geom. Phys. 24, 83 (1998).

[8] H. Friedrich, Spin-2 fields on Minkowski space near space-like and null infinity, Class. Quantum Grav. 20, 101 (2003).

[9] H. Friedrich, Smoothness at null infinity and the structure of initial data, in 50 years of the Cauchy problem in general relativity, edited by P. T. Chruściel \& H. Friedrich, Birkhausser, 2004.

[10] H. Friedrich, Conformal structure of static vacuum data, Comm. Math. Phys. 321, 419 (2013).

[11] H. Friedrich, Peeling or not peeling —is that the question?, Class. Quantum Grav. 35, 083001 (2018).

[12] H. Friedrich \& J. Kánnár, Bondi-type systems near space-like infinity and the calculation of the NP-constants, J. Math. Phys. 41, 2195 (2000).

[13] A. García-Parrado \& J. M. Martín-García, Spinors: a Mathematica package for doing spinor calculus in General Relativity, Comp. Phys. Commun. 183, 2214 (2012).

[14] E. Gasperin \& J. A. Valiente Kroon, Polyhomogeneous expansions from time symmetric initial data, Class. Quant. Grav. 34, 195007 (2017).

[15] E. Gasperin \& J. A. Valiente Kroon, Zero rest-mass fields and the Newman-Penrose constants on flat space, J. Math. Phys. 61, 122503 (2020).

[16] J. Kopiński \& J. A. Valiente Kroon, New spinorial approach to mass inequalities for black holes in general relativity, Physical Review D 103, 024057 (Jan 2021).

[17] R. P. Macedo, M. Minucci \& J. A. Valiente Kroon, The Maxwell-scalar field near spatial infinity, in preparation.

[18] T.-T. Paetz, Killing Initial Data on spacelike conformal boundaries, J. Geom. Phys. 106, 51-69 (2016).

[19] R. Penrose \& W. Rindler, Spinors and space-time. Volume 1. Two-spinor calculus and relativistic fields, Cambridge University Press, 1984. 
[20] R. Penrose \& W. Rindler, Spinors and space-time. Volume 2. Spinor and twistor methods in space-time geometry, Cambridge University Press, 1986.

[21] P. Sommers, Space spinors, J. Math. Phys. 21, 2567 (1980).

[22] J. Stewart, Advanced general relativity, Cambridge University Press, 1991.

[23] L. B. Szabados, Two-dimensional Sen connections and quasi-local energy-momentum, Class. Quantum Grav. 11, 1847 (1994).

[24] L. B. Szabados, Two-dimensional Sen connections in general relativity, Class. Quantum Grav. 11, 1833 (1994).

[25] J. A. Valiente Kroon, On the existence and convergence of polyhomogeneous expansions of zero-rest-mass fields, Class. Quantum Grav. 17, 4365 (2000).

[26] J. A. Valiente Kroon, Polyhomogeneous expansions close to null and spatial infinity, in The Conformal Structure of Spacetimes: Geometry, Numerics, Analysis, edited by J. Frauendiener \& H. Friedrich, Lecture Notes in Physics, page 135, Springer, 2002.

[27] J. A. Valiente Kroon, Does asymptotic simplicity allow for radiation near spatial infinity?, Comm. Math. Phys. 251, 211 (2004).

[28] J. A. Valiente Kroon, A new class of obstructions to the smoothness of null infinity, Comm. Math. Phys. 244, 133 (2004).

[29] J. A. Valiente Kroon, Asymptotic properties of the development of conformally flat data near spatial infinity, Class. Quantum Grav. 24, 3037 (2007).

[30] J. A. Valiente Kroon, Estimates for the Maxwell field near the spatial and null infinity of the Schwarzschild spacetime, J. Hyp. Diff. Eqns. 6, 229 (2009).

[31] J. A. Valiente Kroon, Asymptotic simplicity and static data, 13, 363 (2011).

[32] J. A. Valiente Kroon, Conformal methods in General Relativity, Cambridge University Press, 2016.

[33] J. Valiente Kroon, A rigidity property of asymptotically simple spacetimes arising from conformally flat data, Comm. Math. Phys. 298, 673 (2010).

[34] G. Weinstein, On rotating black holes in equilibrium in general relativity, Communications on Pure and Applied Mathematics 43(7), 903 (1990). 